\title{
Title: Social experience alters different types of learning abilities controlled by distinct brain nuclei in Kryptolebias marmoratus
}

\section{Short title: Social experience and learning ability.}

Authors: Cheng-Yu Li a,b, ${ }^{a}$, Dietmar Kültz ${ }^{\mathrm{c}}$, Audrey K. Ward ${ }^{\mathrm{b}, \mathrm{d}} \&$ Ryan L. Earley ${ }^{\mathrm{b}}$

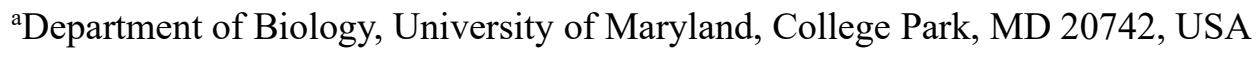

${ }^{\mathrm{b}}$ Department of Biological Sciences, University of Alabama, Tuscaloosa, AL 35487, USA

${ }^{\mathrm{c} D e p a r t m e n t ~ o f ~ A n i m a l ~ S c i e n c e s, ~ U n i v e r s i t y ~ o f ~ C a l i f o r n i a, ~ D a v i s, ~ C A, ~ 95616, ~ U S A ~}$

${ }^{\mathrm{d}}$ Department of Genetics, University of Georgia, Athens, GA, 30602, USA

*Corresponding author: Cheng-Yu Li

Department of Biology, University of Maryland

1210 Biology-Psychology Building, College Park, MD 20742, USA

phone: 205-344-2864

Email: cli12315@umd.edu

Author Contributions: C.Y.L and R.L.E. conceived of and designed the study, analyzed data, and drafted the manuscript; D.K. collected proteomics data and participated in the study design and helped draft the manuscript; C.Y.L. collected behavioral data and collected samples for proteomics analysis; A.K.W. collected, analyzed behavioral data and helped draft the manuscript.

Competing Interest Statement: We have no competing interests.

Classification: Biological Sciences, Ecology

Keywords: winner-loser effects, aggression, spatial learning, risk-avoidance learning, proteomics

\section{This PDF file includes:}

Main Text

Figures 1 to 6 


\section{Abstract}

2 Fighting experiences strongly influence aggressive behavior and physiology (winner-loser

3 effects). These effects are conserved from invertebrates to vertebrates, but the underlying

4 mechanisms remain unclear. Recent studies indicate that the brain social decision-making

5 network (SDN) plays a key role in guiding experience-induced behavioral change. Also, while

6 most studies have focused on how winning and losing experiences alter aggression, growing

7 evidence points to these experiences driving multiple behavioral effects, including changes in the

8 ability to learn. In mangrove rivulus fish (Kryptolebias marmoratus), we discovered that single

9 winning experiences significantly improved spatial learning but not risk-avoidance learning,

10 whereas single losing experiences drove the exact opposite to occur. These results provide strong

11 evidence that winning and losing modulate diverse behaviors served by key nodes within the

12 SDN, specifically the dorsolateral pallium (Dl; fish homolog to mammalian hippocampus, which

13 serves spatial learning) and dorsomedial pallium (Dm; fish homolog to mammalian basolateral

14 amygdala, which responds to fear). We therefore quantified whole-proteome expression within

15 the forebrain (where Dm and Dl are located) of adult rivulus with divergent social experiences.

16 We discovered 23 proteins were significantly differentially expressed in the forebrains of winners

17 and losers. Differentially expressed proteins in losers related to modulation of cellular processes,

18 apoptosis and learning while those in winners related to neuronal plasticity, neuroendocrine 
19 homeostasis, energy utilization, and learning. These results imply that winner-loser effects might

20 be governed by very different patterns of protein expression, which could explain why winners

21 and losers show such pronounced differences in behavioral performance.

\section{Significance Statement}

24 Social interactions permeate the daily lives of most animals and often result in changes in

25 behavior for all parties. This implies that social experiences reorganize the brain in ways that

26 promote the expression of alternative behaviors, or that help individuals cope with the outcome

27 of such interactions. But how do aggressive interactions sculpt the brain at the molecular level?

28 We used an emerging model organism, Kryptolebias marmoratus, to examine whether

29 experiences modulate learning ability and then probe the potential neural mechanisms underlying

30 these behavioral changes. We discovered that single winning and losing experiences dramatically

31 altered spatial learning and risk-avoidance learning, respectively, indicating that winning and

32 losing experiences have markedly different effects on the brain and cognitive processes. 


\section{Introduction}

34 Engaging in aggressive interactions can potently alter physiology and future behavior.

35 Social victory significantly increases aggression (1) and levels of androgenic hormones, like

36 testosterone, in most taxonomic groups, including humans (2-5). On the other hand, social defeat

37 dramatically decreases aggression (1) and can have chronic detrimental effects via prolonged

38 activation of stress-related pathways in brain (6), which can induce depression and a variety of

39 other behavioral disorders $(7,8)$. These responses to prior fighting experiences are termed the

40 'winner effect' and 'loser effect', respectively. Although winner-loser effects are strongly

41 conserved from insects to humans, their underlying neurophysiological mechanisms remain

42 largely unknown. Previous studies, which focus mainly on circulating hormones, indicate that

43 testosterone and its receptor mediate aggression and winner effects $(2,4,9-12)$, but recent studies

44 suggest that other types of neural mechanism, such as activation of dopaminergic system $(13,14)$

45 within social decision-making network (SDN), might also govern behavioral responses to

46 winning (15-17).

47 In addition, while most studies on contest behavior have focused on how winning and

48 losing alter aggression, growing evidence points to these types of experience having manifold

49 behavioral effects, including changes in cognitive ability (18-20). This suggests that social

50 experiences might elicit widespread alterations to brain function. We thus took the approach of 
51 probing the activity of specific brain nuclei by examining the performance of animals on

52 behavioral tasks known to be associated with activation of these brain regions. For instance,

53 spatial learning ability has been associated with prior winning experience (19), which led to the

54 investigating of hippocampus region, whereas the risk-avoidance learning ability has been

55 associated with prior losing experience $(18,20)$, which led to scrutinizing basolateral amygdala.

56 A recent study in fruit flies, Drosophila melanogaster, showed that social defeats induce a long-

57 lasting loser effect associated with de novo protein synthesis (21), which is required for the

58 formation and persistence of long-term memory, whereas social victories had no such long-term

59 behavioral consequences and were not associated with de novo protein synthesis (21). These

60 studies suggest that distinct neurobiological mechanisms might govern loser and winner effects

61 across taxa. However, we know little about the memory-related neurophysiological mechanisms

62 underlying behavioral responses to social competition $(4,22,23)$ or the memory mechanisms

63 that drive persistent changes in behavior following aggressive interactions $(24,25,26)$.

64 Therefore, our first aim was to explore temporal changes in aggression, spatial learning, and risk-

65 avoidance learning in an emerging model organism, mangrove rivulus fish (Kryptolebias

66 marmoratus, hereafter 'rivulus'), following winning and losing experiences. We hypothesized

67 that winners would show increased aggression and proficiency in spatial learning, because

68 selection should favor behavioral processes that facilitate acquisition and defense of a territory 
69 and resources, which may require superior learning capacities in this context. We also

70 hypothesized that losers would show decreased aggression but increased proficiency in risk-

71 avoidance learning because selection should prioritize behaviors that facilitate recovery from

72 aggressive contests, which may require avoiding risk and being less active overall.

73 Traditional social defeat paradigms (7), which expose animals to repeated losing

74 experiences, are useful for identifying the terminal influences of multiple aggressive interactions

75 on behavior and physiology. However, because the brain is exceptionally plastic, it is possible

76 that the neural mechanisms responsible for short-term changes in behavior are different from

77 those that maintain behavioral states over the long-term. While much attention has been given to

78 exploring variation in neurobiology and behavior between animals occupying stable social

79 rankings, there is increased awareness that examining responses to a single win or loss can

80 provide insights into how the brain is initially reorganized by social inputs, and how and whether

81 revamped neural (and associated behavioral) states are maintained over the long-term $(27,28)$.

82 Because fighting experiences likely influence social behavior through a complex array of

83 neuroendocrine interactions, investigating single candidate molecules is not sufficient to

84 understand the mechanisms driving behavioral change. For this reason, and because proteins are

85 ultimately responsible for producing the behavioral phenotype $(29,30)$, the second aim of this

86 study was to quantify protein abundance in rivulus' forebrain, which includes several brain 
87 nuclei (e.g., D1 - fish homolog of mammalian hippocampus; Dm - fish homolog of mammalian

88 basolateral amygdala) implicated in modulating both aggression and learning, as well as

89 physiological responses to acute social experiences. We hypothesized that winning and losing

90 experiences would result in divergent, perhaps unique, patterns of forebrain proteome

91 expression.

\section{Results}

To investigate whether, and for how long, social experiences affect aggression and learning,

95 we conducted a full factorial experiment with 3 experience and 3 decay-time levels. Fish $(n=$

96 675) were allocated to three experience treatments (W: winner; L: loser; N: no fighting

97 experience [control]) and individuals in each experience treatment were subdivided into those

98 exposed to the aggression, spatial learning or risk-avoidance learning tests (Fig. 1A-C, SI

99 Appendix, Movie S1-S5). Animals were subjected to behavioral tests before (on Day 1; pre-

100 experience behaviors) and $1 \mathrm{~h}, 3 \mathrm{~h}$ or $48 \mathrm{~h}$ after social experience (on Day 15-17, based on pre-

101 assigned decay-times; post-experience behaviors). Forebrain proteome expression was quantified

102 in 12 additional individuals $1 \mathrm{~h}$ after winning $(n=4)$, losing $(n=4)$ or control $(n=4)$

103 experiences, the time point at which fish showed pronounced changes in aggression and learning. 
111 mirror image $(P<0.001$, Fig. 2F) than both winners and controls.

113 image with higher, lower, and similar frequencies, respectively; all comparisons were

114 significantly different $(P<0.001$, Fig. 2G). Losers also took significantly longer to launch the

115 first attack relative to their pre-experience behavior, a change that was significantly greater than

116 that observed for winners or controls $(P<0.001$, Fig. 2C). While these patterns remained similar

117 across decay time points (Fig. 2D, Fig. 2H), there was a significant experience $x$ decay-time

118 interaction for latency to first attack (Table S1). Winners' latency to first attack gradually

119 increased, whereas losers' latency to first attack gradually decreased between $1 \mathrm{~h}$ and $48 \mathrm{~h}$,

120 suggesting that winner-loser effects were most pronounced at $1 \mathrm{~h}$, but slowly decayed after $3 \mathrm{~h}$.

121 Also, there were significant differences among lineages in pre-experience aggression, post- 
122 experience aggression, and changes in aggressive behavior (SI Appendix, Fig. S1A, S1B, Table

123 S1).

124

125 Social experience and spatial learning

Individuals assigned to each treatment exhibited similar spatial learning performance before

127 social experiences were obtained (Fig. 3A, 3D, SI Appendix, Table S2). Winners were more

128 likely to pass the spatial learning test than controls at each post-experience time point but not

129 significantly so at $3 \mathrm{~h}$ (overall: $\chi^{2}=11.14, P=0.002 ; 1 \mathrm{~h}: \chi^{2}=4.76, P=0.029 ; 3 \mathrm{~h}: \chi^{2}=1.64, P=$

$130 \quad 0.205$; 48h: $\chi^{2}=6.08, P=0.014$, Fig. 3B, 3C). Winners also completed the learning task more

131 quickly than losers at each post-experience time point, although they did not perform

132 significantly better at $3 \mathrm{~h}$ (losers vs. winners - overall: $P<0.001 ; 1 \mathrm{~h}: P=0.001 ; 3 \mathrm{~h}: P=0.251$;

133 48h: $P=0.039$, Fig. 3E, 3F, SI Appendix, Table S2). Most importantly, winners improved upon

134 their pre-experience performance whereas losers showed virtually no change at all; the difference

135 between winners and losers was significant at all time points except $3 \mathrm{~h}$ (losers vs. winners -

136 overall: $P=0.001$; 1h: $P=0.012 ; 3 \mathrm{~h}: P=0.237$; 48h: $P=0.009$, Fig. 3G, 3H, SI Appendix,

137 Table S2). There was no significant experience $\mathrm{x}$ decay-time interaction on spatial learning,

138 suggesting that performance differences between winners, losers and controls were preserved

139 across time. Lastly, there were significant differences among lineages in pre-experience and post- 
140 experience spatial learning performance but not the change in spatial learning performance $(\boldsymbol{S I}$

141 Appendix, Fig. S1C, S1D; Table S2).

\section{Social experience and risk-avoidance learning}

143 Prior to obtaining social experiences, individuals in the different treatments showed similar

144 performance in the risk-avoidance learning task (Fig. 4A, 4D, SI Appendix, Table S3). Losers

145 had a higher probability of passing the risk-avoidance learning test than controls at each post-

146 experience time point but not significantly so at $3 \mathrm{~h}$ (overall: $\chi^{2}=13.88, P<0.001 ; 1 \mathrm{~h}: \chi^{2}=9.14$,

$147 P=0.003$; 3h: $\chi^{2}=2.13, P=0.145 ; 48 \mathrm{~h}: \chi^{2}=9.14, P=0.003$, Fig. 4B, 4C). Losers also solved

148 the learning task faster than winners at all post-experience time points, but the effect was less

149 pronounced at $48 \mathrm{~h}$ (losers vs. winners - overall: $P<0.001 ; 1 \mathrm{~h}: P=0.001 ; 3 \mathrm{~h}: P=0.025 ; 48 \mathrm{~h}: P$

$150=0.174$, Fig. 4E, 4F, SI Appendix, Table S3). Losers improved upon their pre-experience risk

151 learning performance to a greater extent than both winners and controls at each post-experience

152 time point, although the comparison between winners and losers at $48 \mathrm{~h}$ was not significant

153 (losers vs. winners - overall: $P<0.001$; $1 \mathrm{~h}: P=0.001$; $3 \mathrm{~h}: P=0.037$; 48h: $P=0.121$; losers vs.

154 controls - overall: $P=0.002 ; 1 \mathrm{~h}: P=0.018$; 3h: $P=0.037$; 48h: $P=0.061$; Fig. 4G, 4H, SI

155 Appendix, Table S3). There was no significant experience x decay-time interaction on risk-

156 avoidance learning, suggesting that differences in learning between winners, losers, and controls

157 persisted across post-experience time points. There were significant differences among lineages 
158 in pre-experience and post-experience risk avoidance learning performance but not the change in

159 learning performance (SI Appendix, Fig. S1E, S1F, Table S3).

\section{Comparing the effects of social experience on learning abilities}

163 different social experiences, we pooled data across decay-time points within the same experience

164 type. We then re-categorized individuals based on learning performance (pass or fail) before and

165 after social experiences (e.g., pass-pass, pass-fail, fail-pass, fail-fail; Fig. 5). In the spatial

166 learning test, the pattern of behavioral change in losers was similar to controls (for losers, $26.7 \%$

167 improved [fail to pass] and 24\% regressed [pass to fail]; for controls, $26.7 \%$ improved and $18.7 \%$

168 regressed). However, winners showed a significantly different pattern than controls (41.3\%

169 improved but only $1.3 \%$ regressed). Thus, winning dramatically improved spatial learning

170 ability, whereas losing had relatively little effect (Fig. 5A).

171 For risk-avoidance learning, the pattern of behavioral change in winners was similar to

172 controls (for winners, 5.3\% improved and 18.7\% regressed; for controls: $13.3 \%$ improved and

$17314.7 \%$ regressed). However, $13.3 \%$ of losers improved but $0 \%$ regressed, which was

174 significantly different from controls. Losing therefore had a strong effect on risk-avoidance

175 learning ability, whereas winning had relatively little effect (Fig. 5B). 
177 Proteomic responses to social experiences

178 Quantitative proteomics analysis of the forebrain identified 1545 proteins, 23 of which

179 changed significantly in abundance after social experiences were obtained. Of these differentially

180 expressed proteins, four have functions directly related to learning and memory: i) LRRN4 C-

181 terminal-like protein, which is involved in synapse formation, increased 3.9-fold $(P<0.001)$ in

182 losers compared to controls (Fig. 6); ii) $\mathrm{Ca}^{2+} /$ calmodulin-dependent protein kinase type II

183 subunit $\alpha$, which is involved in long-term potentiation (LTP) and synaptic plasticity, increased

184 3.5-fold $(P<0.001)$ in winners compared to controls (Fig. 6); iii) neuromodulin-like protein,

185 which also participates in LTP, increased 2.5-fold $(P<0.001)$ in losers compared to winners

186 (Fig. 6); iv) $\gamma$-adducin-like protein, which is involved in LTP and neural firing, increased 2.5-

187 fold $(P<0.001)$ in winners compared to losers $($ Fig. 6). Winners were also found to have a 6-

188 fold higher relative abundance of creatine kinase B-type (CKB) protein, which participates

189 mainly in energy transduction, than controls and losers. In addition to learning and memory, nine

190 differentially expressed proteins were related to cellular processes; five to neural plasticity; two

191 to cell death and apoptosis; two to energy utilization and one to immune function (Fig. 6).

192 Overall, losing affected forebrain expression of proteins that modulate cellular processes

193 (calcium signaling/binding, second messenger production, neurotransmitter release and 
194 mobilization of synaptic vesicles), decrease neural plasticity, increase apoptosis and cell death,

195 facilitate recovery from energy deficit (e.g., gluconeogenesis), and mediate learning and memory

196 (e.g., LTP and synapse formation). Winning, however, affected forebrain expression of proteins

197 that mediate cellular processes related to restoration of neuroendocrine homeostasis and signal

198 transduction, increased neuronal plasticity, energy utilization, and learning mechanisms.

200 Discussion

201 We showed that single winning or losing experiences drive markedly different behavioral

202 phenotypes. Predictably, winners increased and losers decreased their aggressive behavior,

203 effects that lasted for at least $48 \mathrm{~h}$. However, we also demonstrated that winning and losing have

204 discernably different effects on learning. Unlike experience-induced changes in aggression,

205 where winners and losers showed opposite responses of similar magnitude, the effects of

206 winning and losing on spatial learning and risk avoidance learning were not symmetrical.

207 Winning increased performance in a spatial learning task but had essentially no effects on

208 performance in a risk-avoidance learning task. On the other hand, losing increased risk-

209 avoidance learning but had no effect on spatial learning. Social experiences also induced

210 pronounced forebrain proteomic responses and, while some proteins were differentially regulated

211 in both winners and losers, many of the proteins were differentially expressed in response to only 
212 one type of experience. These data provide evidence that neurobiological responses to winning

213 and losing are not simply 'opposite sides of the same coin' but rather, are quite unique. This

214 might thus explain the different (but not necessarily opposite) behavioral phenotypes of winners

215 and losers, especially with respect to learning, and highlights the fact that probing behavioral

216 endpoints other than aggression can illuminate key distinctions in how the brain processes

217 divergent social experiences.

218 Winner and loser effects were originally defined by changes in aggression after agonistic

219 interactions, and thus most previous research focused on the roles of androgenic hormones and

220 associated receptors in mediating these effects. However, recent studies in invertebrates have

221 revealed that learning and memory may also change in predictable ways with winning and losing

222 experience $(21,31,32)$. For instance, fruit flies (Drosophila melanogaster) can recognize

223 conspecifics, and losers behave differently when encountering familiar versus unfamiliar

224 opponents, suggesting that learning and memory accompany changes in social status (31).

225 Another study further revealed that repeated losing experiences induced a long-lasting loser

226 effect, which can be blocked by inhibiting protein synthesis, suggesting that the formation of a

227 long-term loser effect requires de novo protein synthesis (23). Interestingly, however, repeated

228 winning experience had no such behavioral or physiological consequences in the fruit flies (23).

229 These results imply that different neurobiological mechanisms might govern the expression of 
230 winner and loser effects. In crayfish, Procambarus clarkii, winner effects can last more than 14

231 days, and the loser effect can last about 10 days (32). Dominant individuals injected with a 5-

232 HT1 receptor antagonist failed to show the winner effect, whereas subordinate individuals

233 injected with adrenergic-like octopamine receptor antagonist failed to show a loser effect (32).

234 These results provided additional evidence for winner and loser effects being modulated by

235 different neurobiological mechanisms. Furthermore, monoamines, such as serotonin and

236 octopamine, are involved in the expression of various behaviors, ranging from aggression to

237 learning and memory in a diverse array of species, suggesting that winner-loser effects could be

238 mediated by changes in neural processes related to cognition.

239 Though previous studies have revealed that fighting experience can affect learning and

240 memory (e.g., 19, 20), few studies investigate whether winning and losing experiences influence

241 different types of learning, or the persistence of experience-induced gains and losses in learning

242 ability. Our data revealed that winning and losing not only altered aggression, but also affected

243 spatial and risk-avoidance learning abilities. Temporal patterns of change in aggression and

244 learning also were quite similar, with the effects being most pronounced $1 \mathrm{~h}$ after fights and

245 gradually decreasing at $3 \mathrm{~h}$ and $48 \mathrm{~h}$. We further discovered that winning and losing independently

246 modulated specific types of learning - spatial learning and risk-avoidance learning, respectively.

247 Spatial learning and memory are mediated primarily by the hippocampus, which provides 
248 animals with spatial information of environment and plays important roles in the consolidation of

249 information from short-term memory to long-term memory (33). Risk-avoidance learning is a

250 type of fear conditioning regulated (along with emotional learning) in large part by the amygdala.

251 Both the hippocampus and amygdala also mediate aggressive behavior $(34,35)$. Most studies

252 hypothesize that winner and loser effects are governed by the same molecule or mechanism, such

253 as testosterone. However, this hypothesis is rarely supported. For instance, Oliveira et al. (4)

254 hypothesized that socially-induced changes in androgen levels should be a causal mediator of

255 both winner and loser effects. They discovered that anti-androgen treatment blocked the winner

256 effect but injecting with androgens failed to rescue the loser effect, suggesting that androgens are

257 involved only in the winner effect. Trannoy et al. (23) showed that winner and loser effects decay

258 over different time courses in fruit flies, which led to the idea that different memory mechanisms

259 might underlie their expression. Our data support this idea and further imply that winning

260 experiences might alter protein expression in the hippocampus and enhance spatial learning,

261 whereas losing experiences might alter protein expression in the amygdala and strengthen risk-

262 avoidance learning. That is, winner and loser effects may not only be governed by different

263 neurobiological mechanisms but might also be mediated by different brain nuclei. Together with

264 previous research, our results suggest that winner-loser effects emerge as a consequence of

265 multiple, perhaps independent neurobiological systems regulating behavioral expression. 
266 Because these independent systems can be tuned by the type and intensity of social experience,

267 this is likely to profoundly increase behavioral variation among individuals as experiences

268 accumulate, which can then provide fodder for natural selection. If responses to winning and

269 losing experiences are heritable, as indicated by significant variation among rivulus lineages in

270 experience-induced changes to aggression, this could facilitate the evolution of adaptive neural

271 and behavioral flexibility.

272 Our proteomics data revealed several promising candidate forebrain proteins associated

273 with social behavior, learning and memory. Identification of brain proteins associated with these

274 behaviors propels dissection of the molecular mechanisms underlying winner and loser effects.

275 For example, creatine kinase B-type $(\mathrm{CKB})$ protein, which is expressed in the hippocampus,

276 cerebellum, and choroid plexus (36), was highly upregulated in winners, but not in losers. CKB

277 mainly participates in energy transduction in the central nervous system but, studies in

278 homozygous knockout mice suggests a critical role for CKB in spatial learning; deficient

279 individuals took longer than wild type individuals to complete the Morris water maze (37). In our

280 study, it is thus possible that winning experiences increase expression CKB in the hippocampus,

281 thereby improving spatial learning abilities. Whether increasing expression of CKB would also

282 alter aggression needs further investigation. Another protein, synapsin-2, which plays a major

283 role in generating synapses and in regulating neurotransmitter release, was significantly 
284 upregulated in the forebrains of losers relative to winners. In mice, synapsin-2 is constitutively

285 upregulated in the hippocampus of losers, and its expression is strongly linked with submissive

286 behavior, which is almost uniformly exhibited by animals that experience social defeat (38).

287 Therefore, our results support the idea that losing experiences could upregulate expression of

288 synapsin-2 in the forebrain but, whether expression of synapsin-2 is causally associated with

289 changes in aggression and risk-avoidance learning are unclear.

290 It is important to note that we only quantified protein abundance $1 \mathrm{~h}$ after social experience

291 because we observed the most prominent behavioral changes at this time point. Thus, one

292 possible explanation is that de novo protein synthesis can cause the observed changes in protein

293 abundance in such short period of time. Alternatively, it is much more likely that specific

294 proteins are rapidly degraded in response to certain signals (39). Studies in rodents revealed that

295 memory consolidation (short-term memory traces being converted to long-term memory)

296 requires not only protein synthesis but also protein degradation (40). In other words, rapid

297 changes in protein abundance could be because certain proteins exist in an unstable state that

298 renders them rapidly degradable in response to a particular stimulus, such as winning or losing

299 experiences.

In summary, we have demonstrated that divergent social experiences alter different learning

301 processes that are mediated by distinct brain nuclei, suggesting that winner effects and loser 
302 effects are governed by very different neurobiological mechanisms. We also identified a group of

303 candidate forebrain proteins that might modulate experience-induced changes in behavior.

304 Further experiments that manipulate expression of these candidate proteins in specific brain

305 nuclei (e.g., hippocampus, amygdala and their homologs in other vertebrates) will advance our

306 knowledge about the neural mechanisms underlying experience-induced changes in aggressive

307 behavior and cognitive abilities.

309 Materials and Methods

\section{Study organism}

311 This study used adult hermaphroditic mangrove rivulus, Kryptolebias marmoratus

312 ('rivulus'), from 25 isogenic lineages whose progenitors were wild caught in Belize, the

313 Bahamas, Florida Keys and peninsular Florida. The animals used in this study were two

314 generations removed from field-caught progenitors and were produced via self-fertilization.

315 Individuals were isolated on the day of hatching and kept individually in 1L translucent plastic

316 containers filled with $750 \mathrm{~mL}$ of $25 \mathrm{ppt}$ synthetic seawater (Instant Ocean ${ }^{\circledR}$ ). Each container was

317 labelled with a unique number for individual identification. Fish were maintained at ambient

318 temperature $\left(27 \pm 1^{\circ} \mathrm{C}\right)$ on a $12 \mathrm{~h}$ light: $12 \mathrm{~h}$ dark photoperiod and fed $2 \mathrm{~mL}$ newly hatched brine

319 shrimp (Artemia) nauplii every day. 
321 Providing social experience and quantifying behavior performance

322 To ensure that individuals received their pre-assigned winning or losing experience, they

323 were fought against much smaller/larger (difference $>2 \mathrm{~mm}$ ) standard losers/winners that had

324 lost/won several fights against conspecific opponents (random selection procedure, [1]).

325 In the aggression test, we quantified individuals' aggressive responses using non-reversing

326 mirror-image stimulation (41, Fig. 1A). The latency to initiate aggressive attacks and frequency

327 of aggressive attacks toward mirror image were recorded as aggression indices. In the spatial

328 learning test, individuals were challenged to recall the location of reward (water + brine shrimp

329 nauplii) in a $37 \mathrm{~L}$ tank that contained only a layer of moist sponge $(1 \mathrm{~cm})$ at the bottom (Fig.

330 1B). This apparatus was modified from Chang et al. (19), and was based on the ecologically

331 relevant premise that rivulus jump or crawl across moist land to seek out water in mangrove

332 forests (42). Fish were given two training sessions (30 min for each session) to become familiar

333 with the environment and to learn the location of the petri dish containing the reward (water +

334 brine shrimp nauplii). During the testing phase, fish were allowed to explore the tank for 30 min

335 to locate the correct petri dish from the previous two training sessions. We considered an

336 individual to have passed or failed the test based on whether it succeeded in locating the correct

337 petri dish; we also recorded the latency to complete the task as a measure of individual spatial 
338 learning ability. The risk-avoidance learning test entailed the focal animal being challenged to

339 learn the association between a visual cue (red color, conditioned stimulus [CS]) and an event

340 indicating risk (black corrugated plastic gliding over the tank, unconditioned stimulus [US], Fig.

341 1C). We anticipated that fish would respond to the simulated predator stimulus by seeking shelter

342 and that they would establish an association between red color (CS) and risk signal (US). An

343 individual passed or failed when it sought shelter within 5 min after seeing the red card appear.

344 We also recorded the latency to complete the task as a measure of individual risk-avoidance

345 learning ability. Individuals that failed the spatial learning or risk-avoidance tasks during either

346 training or during the testing phase were also included in the final data set because a failing

347 result was used for comparison between the pre- and post-experience learning performance.

348 Details of each procedure are provided in SI Appendix, Supplementary Material \& Methods.

349 Sample preparation and protein quantitation in forebrain

350 After receiving social experience, rivulus $(n=12)$ were decapitated at $1 \mathrm{~h}$ in accordance

351 with IACUC standards for euthanasia. Brains were dissected, and forebrains were then separated

352 using a razor blade under a dissecting microscope. Tissues were snap-frozen in liquid nitrogen,

353 stored at $-80^{\circ} \mathrm{C}$ and sent to the University of California, Davis to quantify proteome expression.

354 Protein extraction, protein assays and in-solution trypsin digestion were performed following the

355 protocol established by Kültz and colleagues (43). Detailed procedures regarding sample 
356 preparation are provided in SI Appendix, Supplementary Material \& Methods. Protein IDs

357 were mapped to MSMS spectra of particular tryptic peptides using four different search engines,

358 including PEAKS 8.5, Mascot 2.2.7 (Matrix Science, London, UK; version 2.2.07), X!Tandem

359 Alanine (http://www.thegpm.org/tandem/) and Byonic (Protein Metrics, San Carlos, CA, USA;

360 version 2.12). The complete Kryptolebias marmoratus proteome (38,516 proteins), an equal

361 number of decoy entries and common contaminants (porcine trypsin, human keratin) were used

362 as the reference database for all searches. Results from all four search engines were consolidated

363 in Scaffold 4.4 (Proteome Software Inc., Portland, OR, USA) and proteins represented by at least

3642 unique peptides and meeting a protein level FDR $<1.0 \%$ and a peptide level FDR $<0.1 \%$ were

365 considered valid IDs. Label-free quantitative profiling of peptide intensities and calculation of

366 relative protein abundances in each sample was performed with PEAKS 8.5. The PEAKS protein

367 quantitation is based on the Top3 approach, which measures the area under the curve of the three

368 most abundant unique peptides for a particular protein from the LC-MS/MS chromatogram.

369 Relative abundance of a peptide in a sample was normalized against the overall abundance of all

370 peptides in that sample.

371

\section{Statistical Analysis}


374 levels of aggression before and after social experience, and also whether individuals with

375 different experiences showed variation in the degree to which aggression changed from pre- to

376 post-experience. The response variables were: latency to first attack (ln-transformed to achieve

377 normality) and number of attacks in the pre-experience aggression test or post-experience

378 aggression test, as well as changes in latency to first attack and changes in number of attacks (run

379 in separate models). Type of experience (W, N, L) and decay time (1h, $3 \mathrm{~h}, 48 \mathrm{~h})$ were fixed

380 predictor variables. The interaction term, experience $\mathrm{x}$ decay time, was also included in the

381 models. Lineage and standard length of focal individuals were included in the model as

382 covariates.

383 General linear models also examined whether social experience influenced spatial learning

384 and risk-learning abilities. Pre- and post-experience learning behavior, including latency to pass

385 spatial learning and risk learning tasks, and changes in learning abilities were the response

386 variables. Type of experience (W, N, L) and decay time (1h, 3h, 48h) were fixed predictor

387 variables. The interaction term, experience $\mathrm{x}$ decay, time was also included in the models.

388 Lineage and standard length of focal individuals were included in the model as covariates. 
391 HSD tests are presented as $P$-values in parentheses following a description of the differences

392 (Fig. 2, Fig. 3D-H, Fig. 4D-H).

393 Chi-square tests determined whether the probability of successfully passing each learning

394 task differed between winners versus controls and between losers versus controls (Fig. 3A-C,

395 Fig. 4A-C, Fig. 5). JMP (v. 12; SAS Institute, Cary, NC, USA) was used for all statistical

396 analyses involving behavior.

397 Statistical significance for label-free protein quantitation was based on PEAKSQ $-\log _{10}(P-$

398 values), which were calculated using a previously developed algorithm that has been optimized

399 for proteomics data (44). In this study, a significance threshold of $-\log _{10}(P$-values $) \geq 13$ and fold

400 change $\geq 2.0$ were applied. All proteomics data, including raw data, metadata, Scaffold file,

401 peptide and protein identifications, and quantitative data are accessible in public proteomics

402 repositories (MassIVE AC: MSV000082806, ProteomeXchange AC: PXD010729). The Scaffold

403 file and zipped PEAKSQ data are accessible via ftp download from MassIVE (AC:

404 MSV000082806).

405

406 Acknowledgements

407 We thank Caitlin Curtis for assisting in scoring behavior videos. We also thank all members of

408 the Earley laboratory and Kültz laboratory for their comments on the manuscript and their 
409 support on this project. This research was supported by Sigma Xi Scientific Research Honors

410 Society (Grants-in-Aid: G201703158955-1084) and College Academy for Research, Scholarship

411 and Creative Activity (CARSCA) committee at the University of Alabama.

412 


\section{References}

414 1. Y. Hsu, R. L. Earley, L. L. Wolf, Modulation of aggressive behaviour by fighting

415 experience: mechanisms and contest outcomes. Biol. Rev. 81, 33-74 (2006).

416 2. T. O. Oyegbile, C. A. Marler, Winning fights elevates testosterone levels in California mice

417 and enhances future ability to win fights. Horm. Behav. 48, 259-267 (2005).

418 3. M. J. Fuxjager, G. Mast, E. A. Becker, C. A. Marler CA, The 'home advantage' is necessary

419 for a full winner effect and changes in post-encounter testosterone. Horm. Behav. 56, 214-

$420 \quad 219(2009)$.

421 4. R. F. Oliveira, A. Silva, A. V. M. Canario, Why do winners keep winning? Androgen

422 mediation of winner but not loser effects in cichlid fish. Proc. Biol. Sci. 276, 2249-2256

423 (2009).

424 5. J. M. Carré, J. A. Campbell, E. Lozoya, S. M. Goetz, K. M. Welker KM, Changes in

425 testosterone mediate the effect of winning on subsequent aggressive behaviour.

426 Psychoneuroendocrinology 38, 2034-2041 (2013).

427 6. F. Marini et al., Single exposure to social defeat increases corticotropin-releasing factor and

428 glucocorticoid receptor mRNA expression in rat hippocampus. Brain Res. 1067, 25-35

$429 \quad$ (2006).

4307 K. L. Huhman, Social conflict models: can they inform us about human psychopathology? 
Horm. Behav. 50, 640-646 (2006).

432 8. N. A. Troop, S. Hiskey, Social defeat and PTSD symptoms following trauma. Br. J. Clin.

$433 \quad$ Psychol. 52, 365-379 (2013).

434 9. J. M. Carré, P. H. Mehta, Importance of considering testosterone-cortisol interactions in

435 predicting human aggression and dominance. Aggress. Behav. 37, 489-491 (2011).

436 10. M. J. Fuxjager et al., Winning territorial disputes selectively enhances androgen sensitivity

437 in neural pathways related to motivation and social aggression. Proc. Natl. Acad. Sci. U.S.A.

$438 \quad \mathbf{1 0 7}, 12393-12398(2010)$.

439 11. M. J. Fuxjager, T. O. Oyegbile, C. A. Marler, Independent and additive contributions of

440 postvictory testosterone and social experience to the development of the winner effect.

$441 \quad$ Endocrinology 152, 3422-3429 (2011).

442 12. C. Y. Li, R. L. Earley, S. P. Huang, Y. Hsu, Fighting experience alters brain androgen

443 receptor expression dependent on testosterone status. Proc. Biol. Sci. 281, 20141532 (2014).

444 13. E. A. Becker, C. A. Marler, Postcontest blockade of dopamine receptors inhibits

445 development of the winner effect in the California mouse (Peromyscus californicus). Behav.

$446 \quad$ Neurosci. 129, 205-213 (2015).

447 14. J. J. Schwartzer, L. A. Ricci, R. H. Melloni Jr., Prior fighting experience increases

448 aggression in Syrian hamsters: implications for a role of dopamine in the winner effect. 
450 15. S. M. Goetz et al., Testosterone rapidly increases neural reactivity to threat in healthy men:

451 a novel two-step pharmacological challenge paradigm. Biol. Psychiatry 76, 324-331 (2014).

452 16. J. M. Carré, N. A. Olmstead, Social neuroendocrinology of human aggression: examining the role of competition-induced testosterone dynamics. Neuroscience 286, 171-186 (2015).

454 17. R. M. de Almeida, J. C. Cabral, R. Narvaes, Behavioural, hormonal and neurobiological

455 mechanisms of aggressive behaviour in human and nonhuman primates. Physiol. Behav.

143, 121-135 (2015).

18. L. Kaczer, S. Pedetta, H. Maldonado, Aggressiveness and memory: Subordinate crabs present higher memory ability than dominants after an agonistic experience. Neurobiol. Learn. Mem. 87, 140-148 (2007). of winning and losing and the role of hormones. Integr. Comp. Biol. 52, 801-813 (2012). impairs exploratory behaviors and general cognitive abilities. Behav. Brain Res. 232, 294305 (2012). 
Acad. Sci. U.S.A. 113, 4818-4823 (2016).

22. V Kloke et al., The winner and loser effect, serotonin transporter genotype, and the display of offensive aggression. Physiol. Behav. 103, 565-574 (2011).

470 23. K. Hirschenhauser, M. Gahr, W. Goymann, Winning and losing in public: audiences direct future success in Japanese quail. Horm. Behav. 63, 625-633 (2013).

472 24. Y. T. Lan, Y. Hsu, Prior dominance experience exerts a long-term influence on subsequent

473 winner and loser effects. Front. Zool. 8, 28 (2011).

$47425 . \quad$ K. L. Hollis, The role of learning in the aggressive and reproductive behavior of blue 475 gouramis, Trichogaster trichopterus. Environ. Biol. Fishes 54, 355-369 (1999).

476 26. R. E. Carpenter, C. H. Summers, Learning strategies during fear conditioning. Neurobiol. Learn. Mem. 91, 415-423 (2009).

478 27. K. P. Maruska, A. Zhang, A. Neboori, R. D. Fernald, Social opportunity causes rapid transcriptional changes in the social behaviour network of the brain in an African cichlidfish. J. Neuroendocrinol. 25, 145-157 (2013).

481 28. T. Hattori, W. Wilczynski, Differences in forebrain androgen receptor expression in winners and losers of male anole aggressive interactions. Brain Res. 1582, 45-54 (2014).

483 29. C. M. Valcu, B. Kempenaers, Proteomics in behavioral ecology. Behav. Ecol. 26, 1-15 (2015). 
30. C. Vogel, E. M. Marcotte, Insights into the regulation of protein abundance from proteomic and transcriptomic analysis. Nat. Rev. Genet. 13, 227-232 (2012).

31. A. Yurkovic, O. Wang, A. C. Basu, E. A. Kravitz, Learning and memory associated with aggression in Drosophila melanogaster. Proc. Natl. Acad. Sci. U.S.A. 103, 17519-17524 (2006).

32. Y. Momohara, H. Minami, A. Kanai, T. Nagayama, Role of cAMP signalling in winner and loser effects in crayfish agonistic encounters. Eur. J. Neurosci. 44, 1886-1895 (2016).

33. S. McKenzie, H, Eichenbaum, Consolidation and reconsolidation: two lives of memories? Neuron 71, 224-233 (2011). are elevated during aggression: influence of sympathetic social signaling. Brain Res. 870, $170-178(2000)$. coping: a comparative study in mouse and rat selection lines. Brain Behav. Evol. 70, 274285 (2007). 554 (1996). 
503 37. F. Streijger et al., Mice lacking the UbCKmit isoform of creatine kinase reveal slower

504 spatial learning acquisition, diminished exploration and habituation, and reduced acoustic

505 startle reflex responses. Mol. Cell Biochem. 256/257, 305-318 (2004).

506 38. E. Nesher et al., Synapsin IIb as a functional marker of submissive behavior. Sci. Rep. 5, 10287 (2015).

39. F. Yoshizawa, T. Nagasawa, N. Nishizawa, R. Funabiki, Protein synthesis and degradation 1156-1159 (1997).

40. D. Fioravante, J. H. Byrne, Protein degradation and memory formation. Brain Res. Bull. 85,

513 41. C. Y. Li, C. Curtis, R. L. Earley, Nonreversing mirrors elicit behaviour that more accurately predicts performance against live opponents. Anim. Behav. 137, 95-105 (2018).

515 42. A. J. Pronko, B. M. Perlman, M. A. Ashley-Ross, Launches, squiggles and pounces, oh my!

516 The water-land transition in mangrove rivulus (Kryptolebias marmoratus). J. Exp. Biol.

$517 \quad 216,3988-3995$ (2013).

518 43. D. Kültz, J. Li, A. Gardell, R. Sacchi, Quantitative molecular phenotyping of gill remodeling in a cichlid fish responding to salinity stress. Mol. Cell. Proteomics 12, 39623975 (2013). 
521 44. J. Cox, M. Mann, MaxQuant enables high peptide identification rates, individualized p.p.b.-

522 range mass accuracies and proteome-wide protein quantification. Nat. Biotechnol. 26, 1367-

$523 \quad 1372(2008)$.

524 


\section{Figures}

(A)

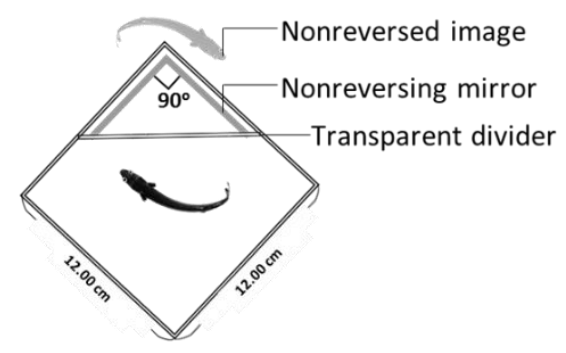

(B)

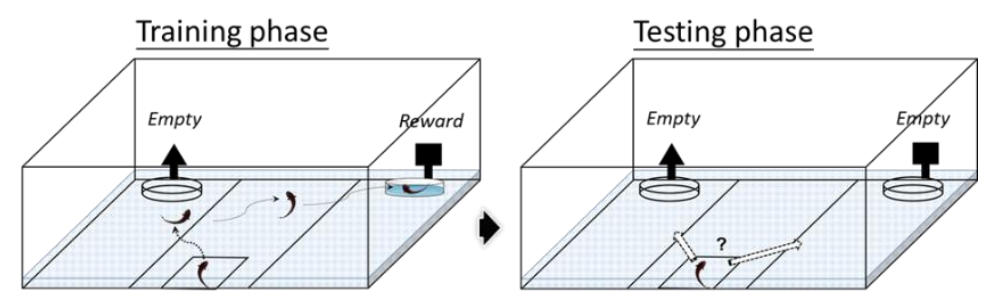

(C)
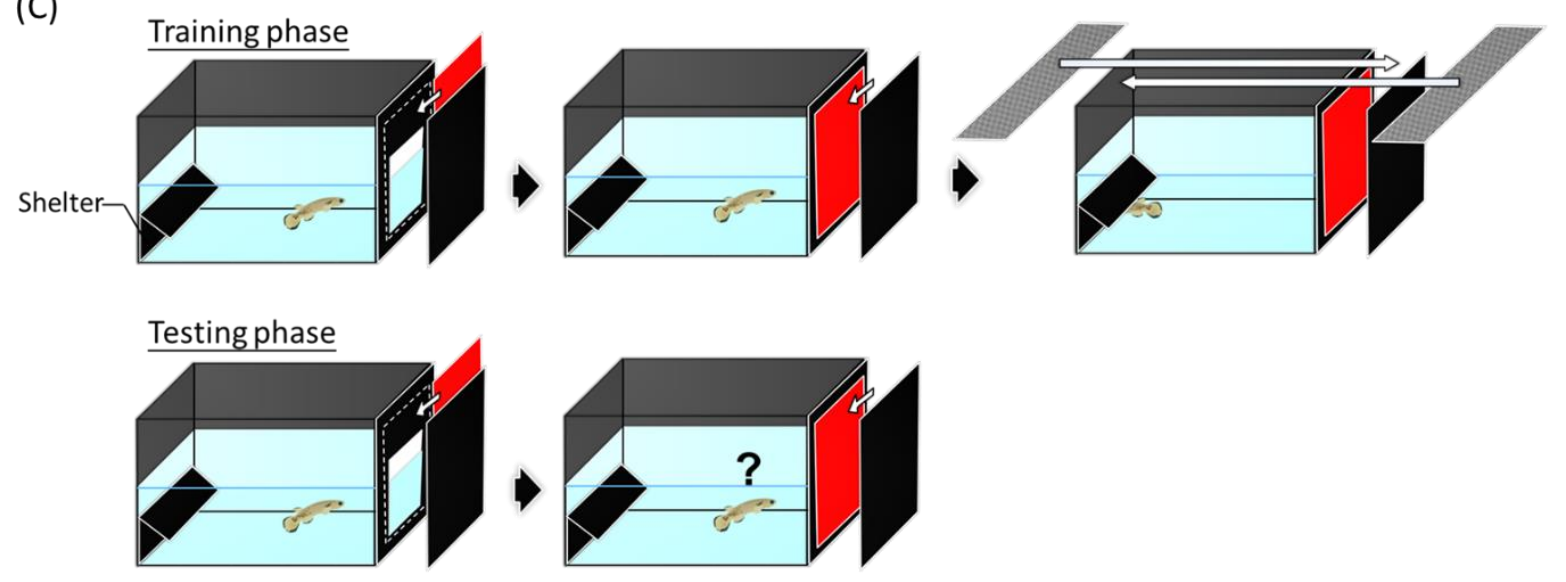

528 Figure 1. Setups for the (A) aggression test (non-reversing mirror image stimulation) (B) spatial

529 learning test, which challenged fish to navigate an arena to find a reward (water/food) (C) risk530 avoidance learning test, which challenged fish to associate red color with risk. 

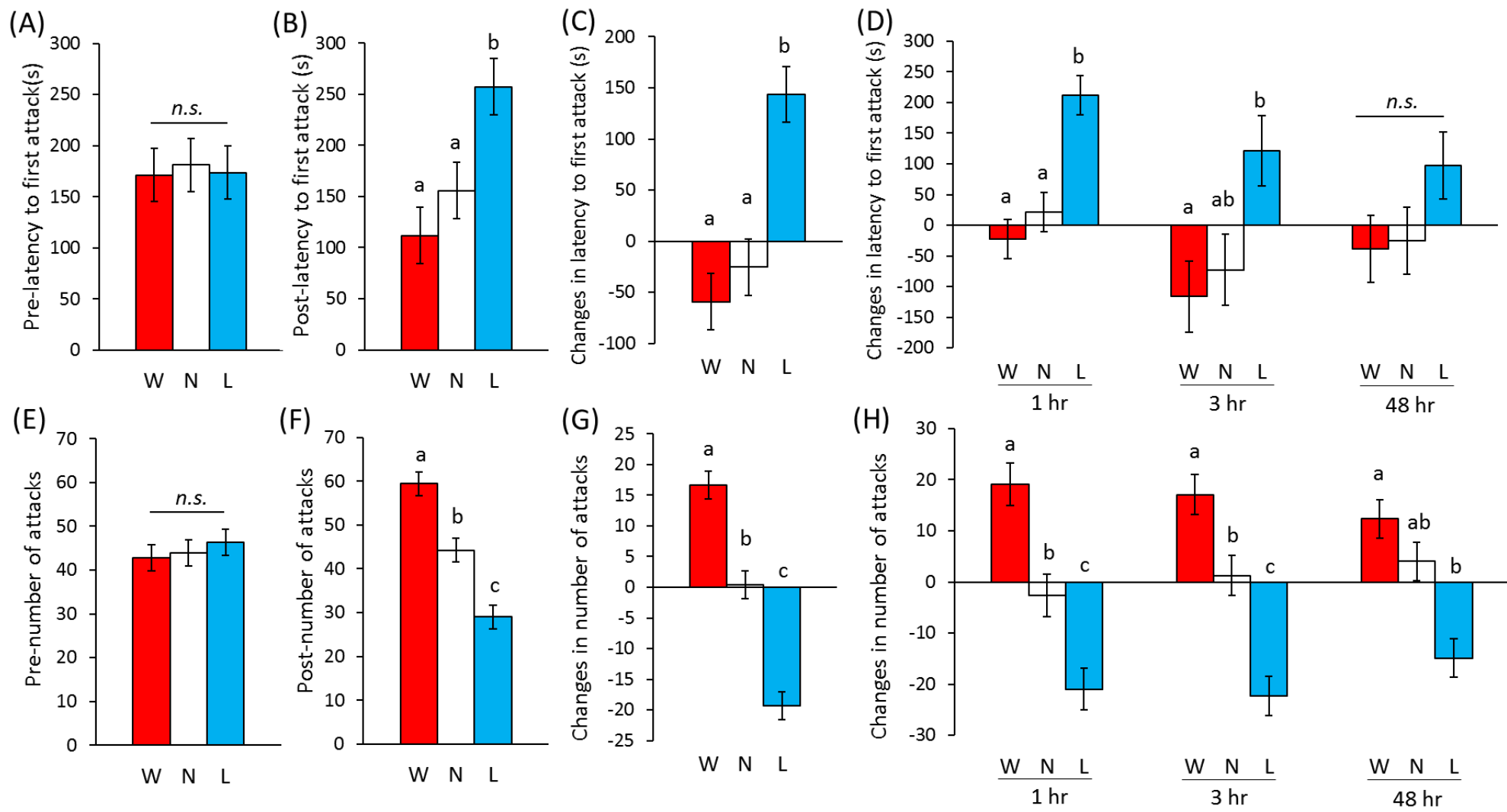

532 Figure 2. The influence of social experience on aggression. Differences among treatments in aggressive responses toward the non-reversing mirror image: $(\mathbf{A})(\mathbf{E})$ before social experience, and $(\mathbf{B})(\mathbf{F})$ after social experience. Differences among treatments in how the animals responded to social experience (post-experience behavior minus pre-experience behavior), with respect to (C) latency to first attack and (G) number of attacks towards the mirror image. Temporal changes in winner-loser effects for (D) latency to first attack and (H) number of attacks from $1 \mathrm{~h}, 3 \mathrm{~h}$ to $48 \mathrm{~h}$ post-experience. Note that 'latency to first attack' is inversely related to aggression such that negative changes in latency to first attack indicate increased aggression. Different lowercase letters indicate significant differences between treatments within a given histogram plot (Tukey's HSD, $P<0.05$; n.s. non-significant; W-winners, N-control (no) experience, L-losers; $n=75$ for each experience). 
bioRxiv preprint doi: https://doi.org/10.1101/2021.04.25.441338; this version posted April 26, 2021. The copyright holder for this preprint (which was not certified by peer review) is the author/funder. All rights reserved. No reuse allowed without permission.

(A)

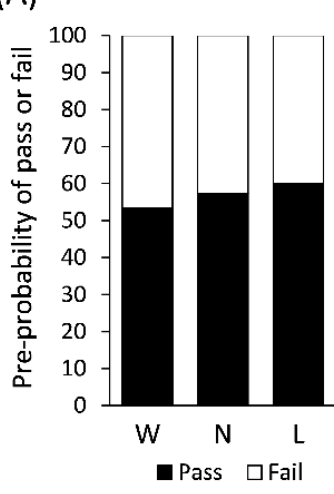

(D)

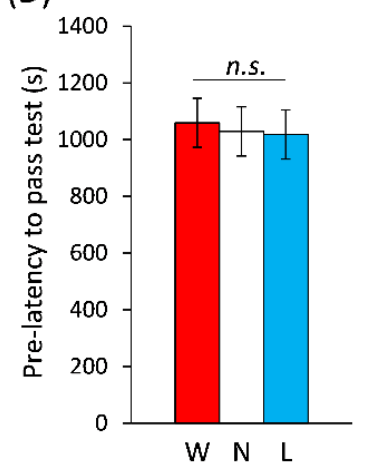

(G)

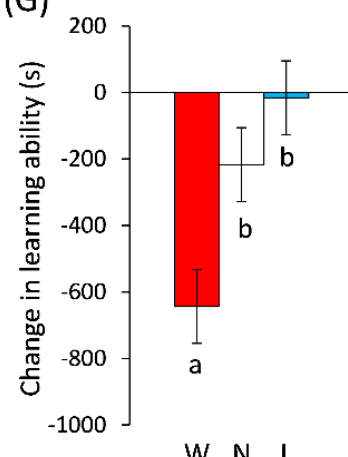

(B)

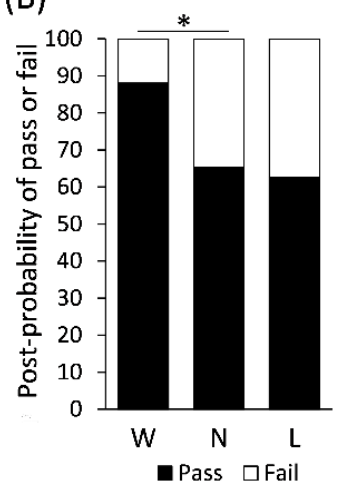

(E)

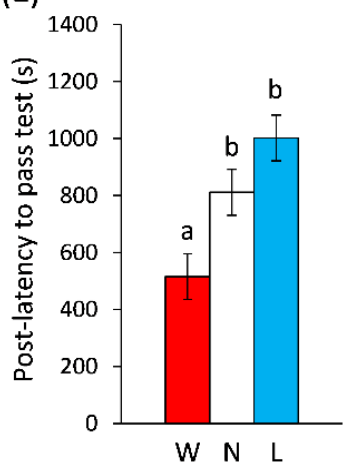

(C)

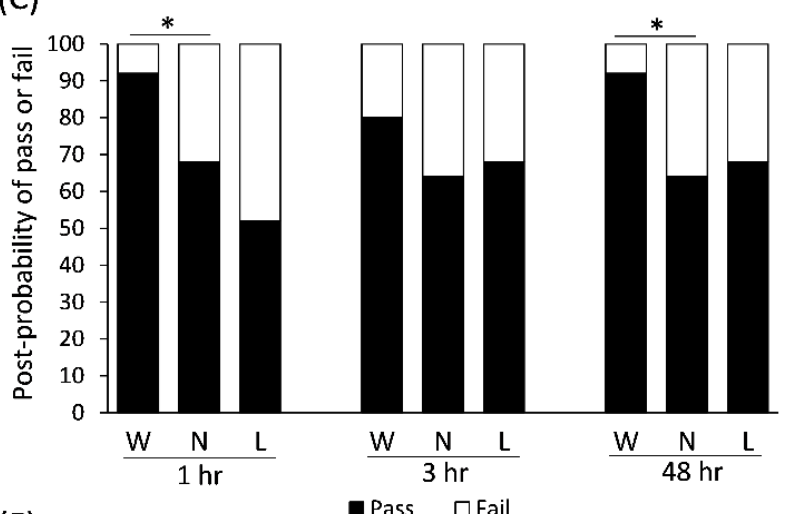

(F)

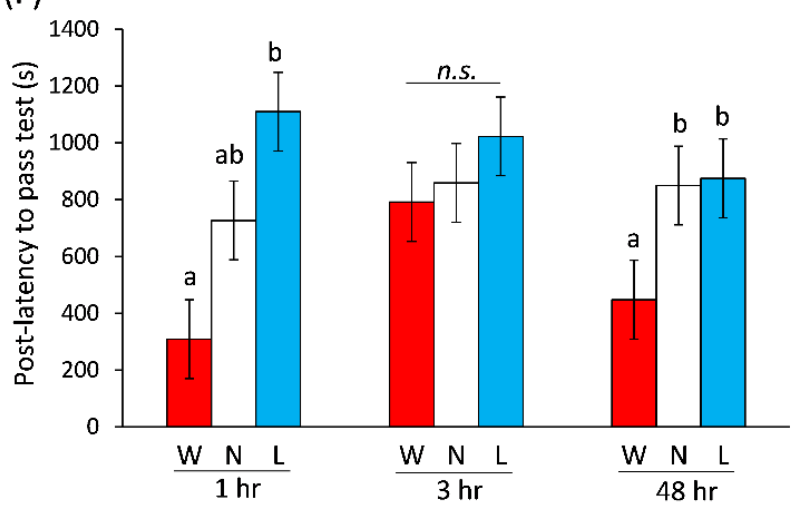

539 Figure 3. The influence of social experience on spatial learning. Differences among

540 treatments prior to social experience for (A) probability of passing/failing the learning test

541 and (D) latency (in seconds, $s$ ) to complete the learning test. Effects of social experience on

542 spatial learning, including (B) probability of passing/failing the test, (E) latency to complete

543 the learning test and (G) change in learning ability (post-experience minus pre-experience

544 performance). Temporal changes in experience effects on spatial learning behavior from $1 \mathrm{~h}$,

$5453 \mathrm{~h}$ to $48 \mathrm{~h}$, including (C) probability of passing/failing the test, (F) latency to complete the

546 learning test and $\mathbf{( H )}$ change in learning ability. Note that 'latency to pass the learning test' is

547 inversely correlated with learning ability such that negative changes in latency indicate

548 increased learning performance. Asterisk indicates significant difference between treatments

549 (Chi-square test). Different lowercase letters indicate significant differences between

550 treatments within a histogram plot (Tukey's HSD, $P<0.05$; n.s. non-significant; W-winners,

551 N-control (no) experience, L-losers; $n=75$ for each experience). 
bioRxiv preprint doi: https://doi.org/10.1101/2021.04.25.441338; this version posted April 26, 2021. The copyright holder for this preprint (which was not certified by peer review) is the author/funder. All rights reserved. No reuse allowed without permission.

(A)

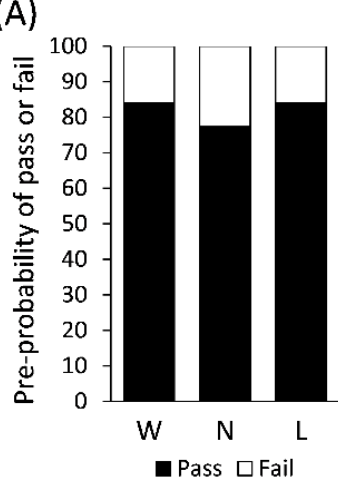

(D)

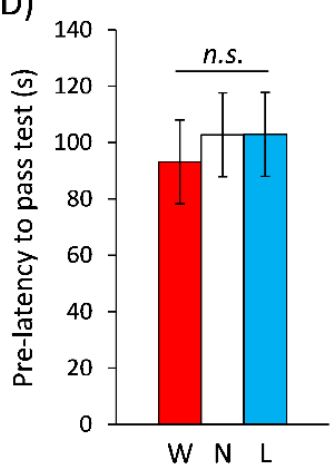

(G)

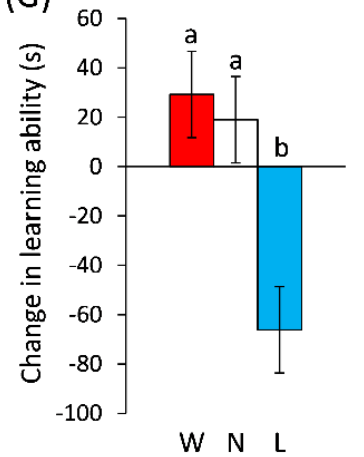

(B)

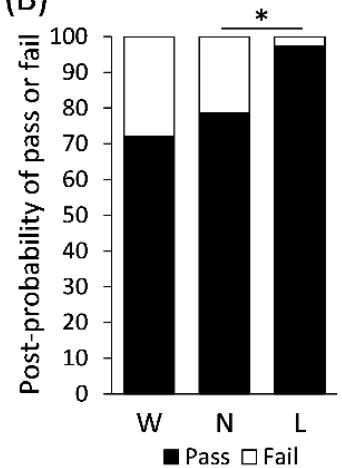

(E)

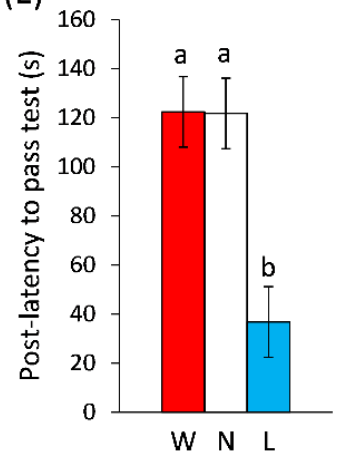

(H)
(C)

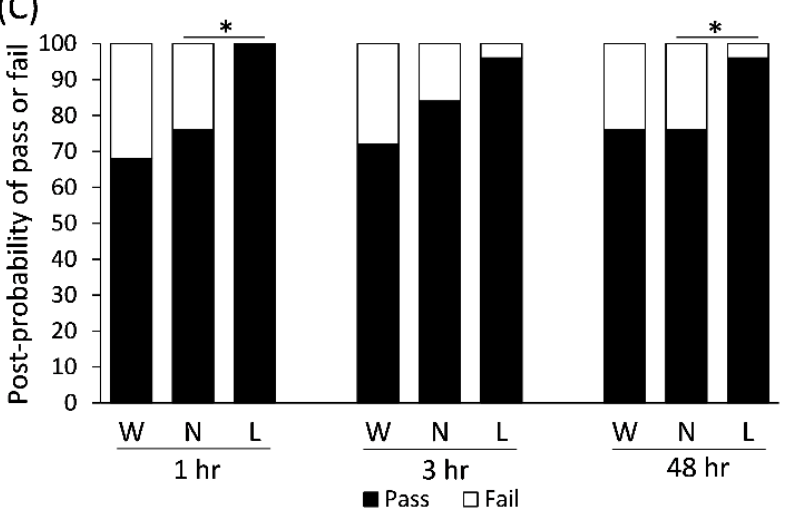

(F)

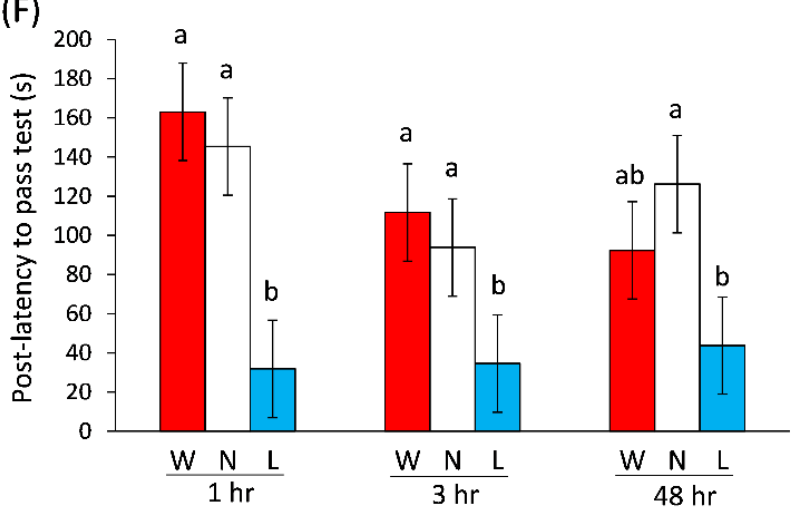

552 Figure 4. The influence of social experience on risk-avoidance learning. Differences among

553 the treatments prior to social experience for (A) probability of passing/failing the learning test

554 and (D) latency (in seconds, $s$ ) to complete the learning test. Effects of social experience on

555 risk-avoidance learning, including (B) probability of passing/failing the test, (E) latency to

556 complete the learning test, and $(\mathbf{G})$ change in learning ability (post-experience minus pre-

557 experience performance). Temporal changes in experience effects on spatial learning

558 behavior from 1h, 3 h to $48 \mathrm{~h}$, including (C) probability of passing/failing the tests, (F) latency

559 to complete the learning test and $(\mathbf{H})$ change in learning ability. Note that 'latency to pass

560 learning test' is inversely correlated with learning ability such that negative changes in

561 latency indicate increased learning performance. Asterisk indicates significant difference

562 between treatments (Chi-square test). Different lowercase letters indicate significant

563 differences between treatments within a histogram plot (Tukey's HSD, $P<0.05$; n.s. non-

564 significant; W-winners, N-control (no) experience, L-losers; $n=75$ for each experience). 
(A)

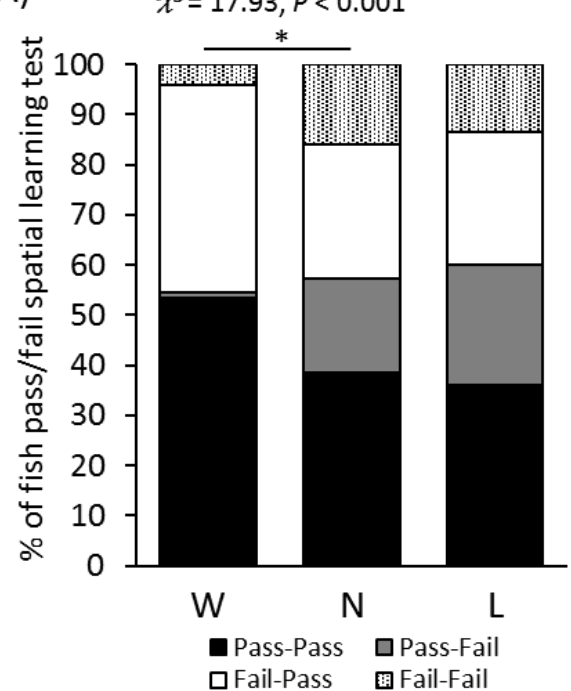

(B)

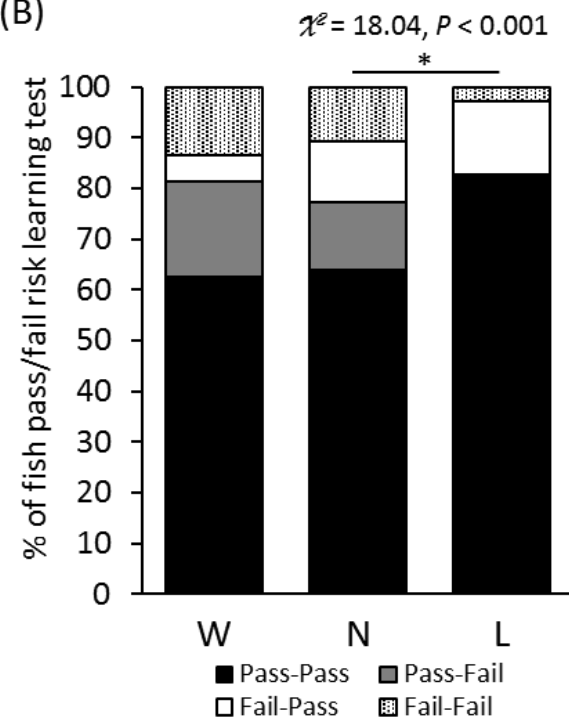

566

567 Figure 5. Comparison of probabilities for consecutive pass-fail sequences among the three

568 treatment groups in (A) spatial learning ability and (B) risk-avoidance learning ability. (Pass-

569 Pass: individuals passed both pre- and post-experience learning tests; Pass-Fail: individuals

570 passed pre-experience learning test but failed post-experience learning test; Fail-Pass:

571 individuals failed pre-experience learning test but passed post-experience learning test; Fail-

572 Fail: individuals failed both pre- and post-experience learning tests; W-winners, N-control

573 (no) experience, L-losers; $n=75$ for each experience). Asterisk indicates significant

574 difference between treatments (Chi-square test).

575 


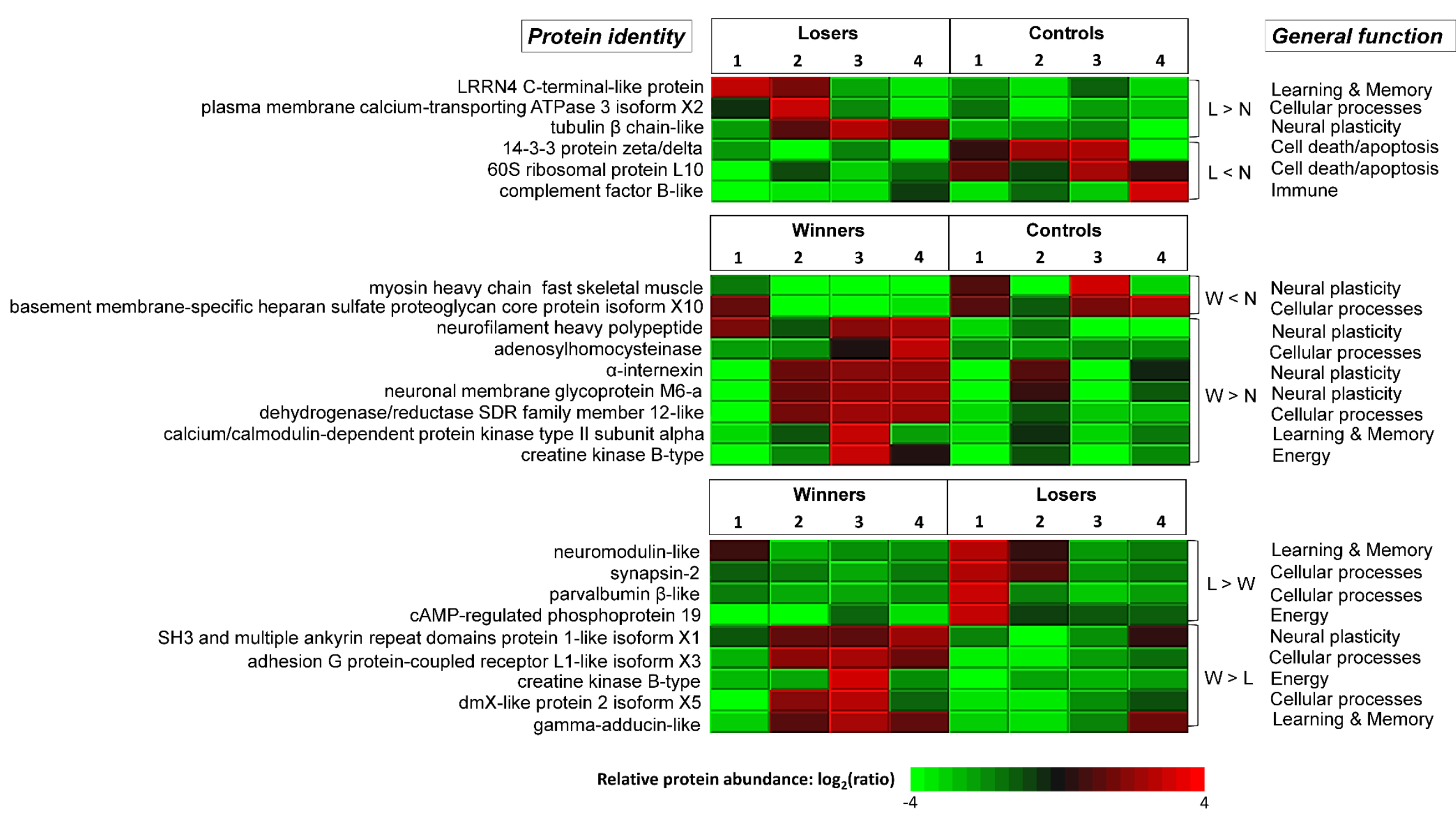

576 Figure 6. Relative abundances of forebrain proteins that were significantly up- or down-regulated after social experience. Each column

577 represents a single individual (winner $n=4$, loser $n=4$, control $n=4$ ) and each row represents a unique protein. Red rectangles represent

578 proteins that showed increased expression after social experience and green rectangles represent proteins that showed decreased expression after

579 social experience. Note that the heat map color is based on a $\log _{2}$ scale. (W-winners, N-control (no) experience, L-losers). 